\title{
Analytical Procedure Precision
}

National Cancer Institute

\section{Source}

National Cancer Institute. Analytical Procedure Precision. NCI Thesaurus. Code C134243.

The closeness of agreement (degree of scatter) between a series of measurements

obtained from multiple sampling of the same homogeneous sample under the prescribed conditions. Precision may be considered at three levels: repeatability, intermediate precision and reproducibility. 